\title{
Computer model of high-precision navigation of a small satellite constellation for remote Earth sensing tasks
}

\author{
Valeriy Tutatchikov ${ }^{1 *}$, Oleg Drozd ${ }^{1}$ и Denis Kapulin ${ }^{1}$ \\ ${ }^{1}$ Siberian Federal University, School of Space and Information Technology, 660074, Krasnoyarsk, \\ Russian Federation
}

\begin{abstract}
One of the key tasks in the formation of the small satellite constellation is to ensure its stable configuration in time. The specific of this problem lies in the fact that the task must be solved in real time with sufficiently high accuracy with significant restrictions on the power consumption of onboard satellite systems. The paper briefly discusses one possible approach of secondary signal processing and solving the navigation and ballistic tasks, which involves the joint use of code, phase measurement methods, integer phase ambiguity resolution, and intersatellite measurements.
\end{abstract}

\section{Introduction}

It can be argued that by now conditions have emerged for the creation and deployment of fully functional space systems based on orbital constellations of of the small satellite (SST), which are considered not as an alternative to existing systems based on medium and heavy spacecraft, but as an addition, significantly expanding the solution applied tasks in various fields of activity.

SST open up wide opportunities for the synthesis of space systems with new qualitative capabilities. For example, for space systems of Earth remote sensing (ERS), the following systemic effects are possible:

1. Multi-position, multi-range and simultaneous observation of an object from several SSTs, providing the effect of stereo photography.

2. Possibility of integrating information received from heterogeneous onboard complexes of special equipment of SST, leading to an increase in the information content of observation.

3. Ensuring the continuity of observation due to the possibility of "passing the baton" of observation to other SST of the orbital constellation that are sequentially entering the object's visibility zone.

The use of SST for remote sensing purposes has great prospects and can significantly improve the consumer qualities of space information, but at the same time it requires solving a number of scientific and technical problems. Along with the system-wide tasks of

\footnotetext{
* Corresponding author: vtutatchikov@mail.ru
} 
maintaining the integrity of the orbital constellation, it is required to solve the tasks of autonomous navigation-ballistic and frequency-time support [1].

At present, for autonomous navigation support of autonomous small satellite, onboard navigation systems based on optical-electronic sensors of celestial bodies (Earth, Sun, navigation stars), inertial attitude control systems, multichannel receivers of signals from global navigation satellite systems (GNSS) are used [3-5]. For ground support of small satellite operation, networks of ground posts for radar and optical control of the current satellite position are used [6-9]. One of the key disadvantages of the existing control facilities is their dependence on the ground segment of the group control and the presence of gaps in the navigation field associated with the territorial location of control posts. In this paper, we propose a solution to the problem of high-precision navigation and ballistic support of a large group of SST in autonomous mode without the use of ground facilities and with minimization of the cost of the proposed solutions [2].

\section{Method description}

The proposed methodology for the navigation support of the SST constellation is a new combination of known methods of secondary processing of navigation information received from GNSS GPS and GLONASS. Solving the navigation problem by the onboard means of the small spacecraft in autonomous mode includes the following procedures:

1) individual and group calculation of the absolute and relative coordinates of the SST by means of code and phase measurements $[10,11]$;

2) calculation of the motion parameters of the center of mass of the small satellite (components of the velocity vector) based on the results of the assessment of pseudovelocities in the code and phase modes;

3) solution of the integer ambiguity of phase measurements of pseudo-range by methods based on the use of second phase differences, calculated using the first phase differences $[12,13]$

4) provision of mutual navigation measurements by means of onboard equipment for inter-satellite measurements and inter-satellite communication lines [14, 15].

\section{Experimental results}

To test the theoretical principles of the implementation of these procedures as applied to a constellation of small satellite with a minimum composition: the master (leader) and the slave satellites, a computer model of navigation support was developed. The simulation model is implemented in $\mathrm{C}++$ in the $\mathrm{Qt}$ development environment. The results of calculating the absolute coordinates and speed of the master small spacecraft are shown in Figure 1, relative coordinates and speed of the slave small spacecraft - in figure 2.

The simulation results showed that the simulation model of the autonomous navigation support of the small satellite constellation meets the requirements for solving the navigation-ballistic problem, in particular, in terms of the root-mean-square error in measuring relative coordinates - no more than $0.02 \mathrm{~m}$ and relative speed - no more than $0.01 \mathrm{~m} / \mathrm{s}$ (figure 3). 


\section{RPERS 2020}

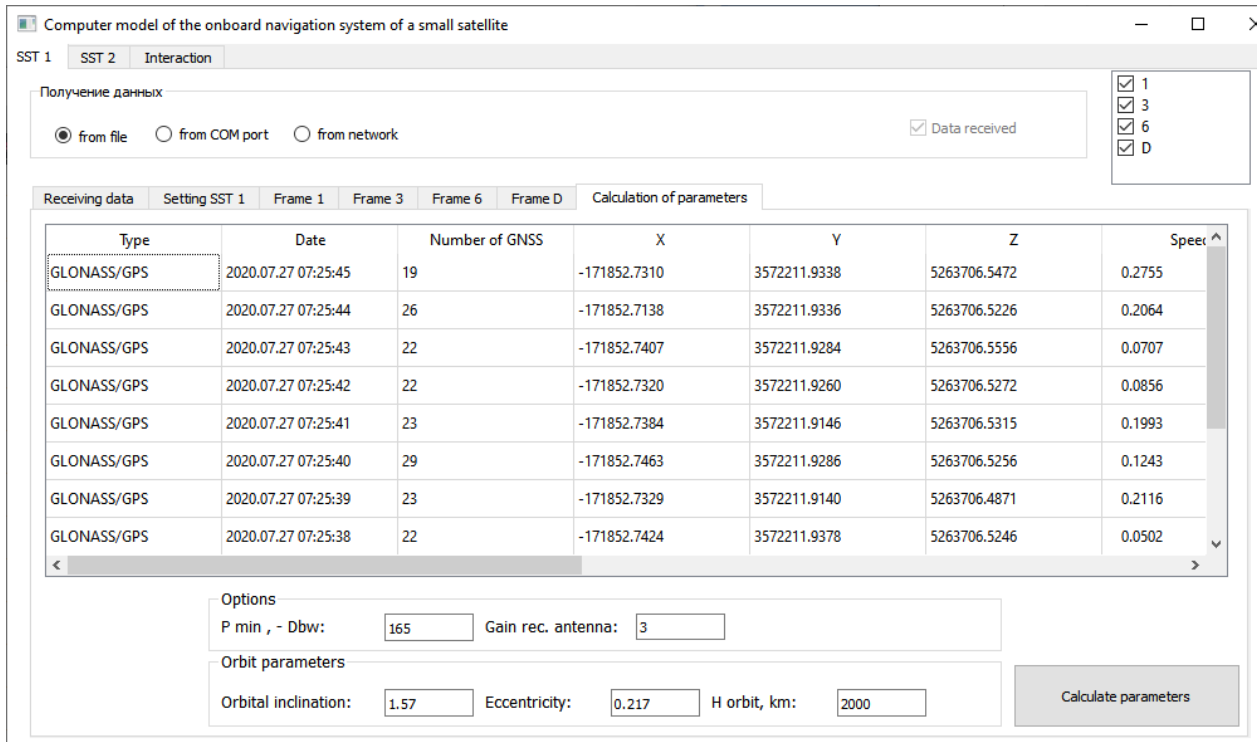

Fig. 1. The absolute coordinates and speed of the leading SST

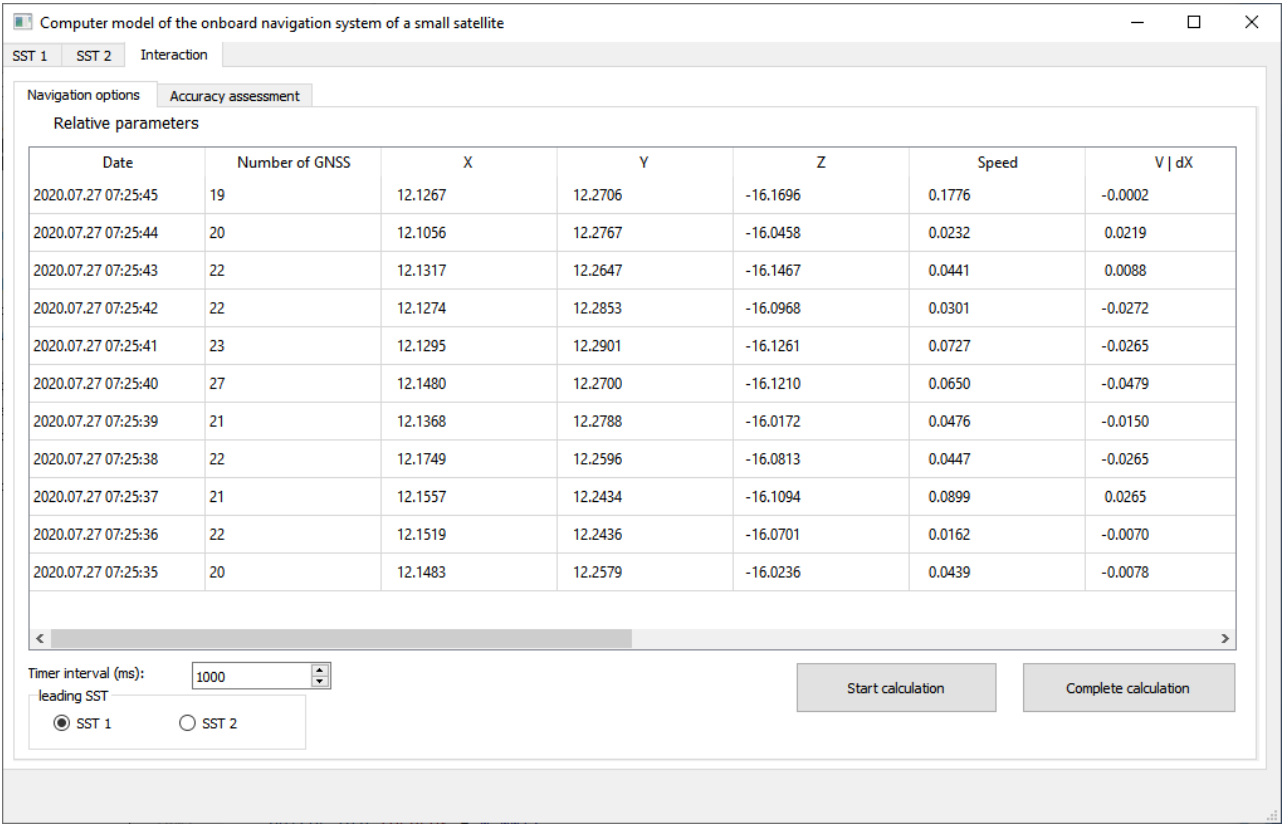

Fig. 2. Relative coordinates and speed of the slave SST 


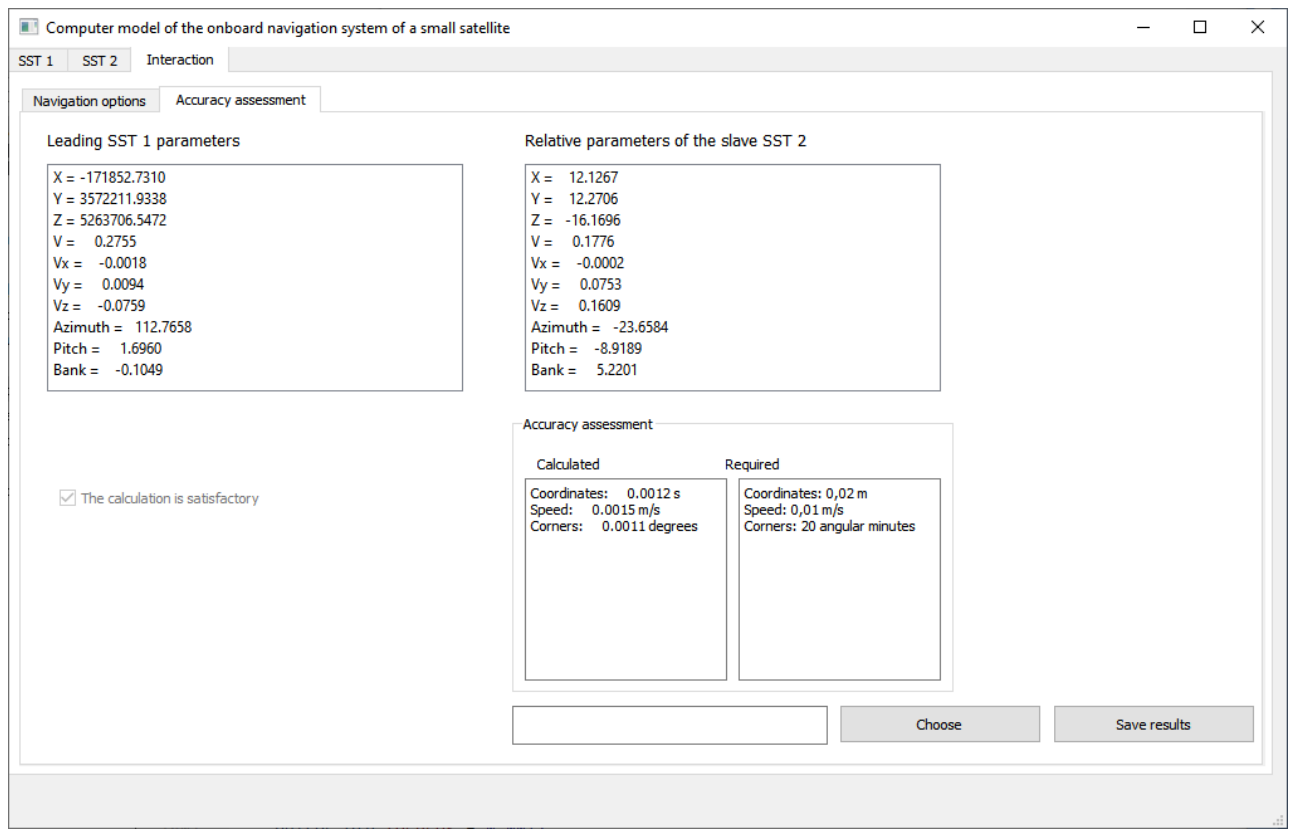

Fig. 3. Estimation of the accuracy of calculating the coordinates and speed of the slave SST

\section{Conclusion}

The development of methods and means of navigation support for high-precision autonomous navigation of constellation of small satellite for performing the tasks of remote sensing of the Earth involves the integration of various methods of processing navigation signals. The paper briefly considers one of the options for such a complex procedure for the secondary processing of navigation signals and the solution of the navigation-ballistic problem, involving the combined use of methods of code, phase measurements, solution of integer phase ambiguity, and intersatellite measurements.

\section{Acknowledgments}

The work was supported by the Ministry of Education and Science of the Russian Federation (Agreement № 075-15-2019-1684, unique ID project RFMEFI60819X0274).

\section{References}

1. J.R. Kopacz, R. Herschitz and J . Roney, Acta Astronaut, 170, 93-105 (2020)

2. X. Ma, J. Fang and X. Ning, Prog. Aerosp. Sci., 63, 56-66 (2013)

3. X. Sun, C. Han and P. Chen, Aerosp. Sci. Technol., 67, 228-36 (2017)

4. D.D. Dmitriev, V.N. Tyapkin, Y.L. Fateev, A.B. Gladyshev and P.Y. Zverev, Moscow Workshop on Electronic and Networking Technologies (MWENT), 1-5 (2020)

5. G. Giorgi, T.D. Schmidt, C. Trainotti, R. Mata-Calvo, C. Fuchs, M.M. Hoque, J. Berdermann, J. Furthner, C. Günther, T. Schuldt, J. Sanjuan, M. Gohlke, M. Oswald, C. Braxmaier, K. Balidakis, G. Dick, F. Flechtner, M. Ge, S. Glaser, R. König, G. 
Michalak, M. Murböck, M. Semmling and H. Schuh, Adv. Sp. Res., 64, 1256-73 (2019)

6. S. Sgubini and G.B. Palmerini, IEEE Aerospace Conference, 1-7 (2010)

7. Y. Liu, Y. Chen, Y. Jiao, H. Ma and T. Wu, IEEE Access, 8, 63923-34 (2020)

8. K. Riesing, H. Yoon and K. Cahoy, IEEE International Conference on Space Optical Systems and Applications (ICSOS), 108-14 (2017)

9. D. Strugarek, K. Sośnica and A. Jäggi, Adv. Sp. Res., 63, 417-31 (2019)

10. P. Teunissen and O. Montenbruck, Springer International Publishing (2017)

11. A. Oxley, A Mathematical Discourse ed Academic Press (2017)

12. A. Raskaliyev, S.H. Patel, T.M. Sobh and A. Ibrayev, IEEE Access, 8, 24873-86 (2020)

13. D.D. Dmitriev, Y.L. Fateev and V.N. Ratushniak, International Siberian Conference on Control and Communications (SIBCON), 1-4 (2019)

14. A.M. Crisan, A. Martian, R. Cacoveanu and D. Coltuc, Measurement, 154, 107479 (2020)

15. F. Yu, Z. He and N. Xu, Acta Astronaut., 160, 646-55, (2019) 